\title{
Toward semiclassical theory of quantum level correlations of generic chaotic systems.
}

\author{
Daniel L. Miller \\ Dept. of Physics of Complex Systems, \\ The Weizmann Institute of science, Rehovot, 76100 Israel \\ e-mailfndaniil@wicc.weizmann.ac.il
}

(November 30, 1997)

\begin{abstract}
In the present work we study the two-point correlation function $R(\varepsilon)$ of the quantum mechanical spectrum of a classically chaotic system. Recently this quantity has been computed for chaotic and for disordered systems using periodic orbit theory and field theory. In this work we present an independent derivation, which is based on periodic orbit theory. The main ingredient in our approach is the use of the spectral zeta function and its autocorrelation function $C(\varepsilon)$. The relation between $R(\varepsilon)$ and $C(\varepsilon)$ is constructed by making use of probabilistic reasoning similar to that which has been used for the derivation of the Hardy - Littlewood conjecture. We then convert the symmetry properties of the function $C(\varepsilon)$ into relations between the so-called diagonal and the off-diagonal parts of $R(\varepsilon)$. Our results are valid for generic systems with broken time reversal symmetry, and with non-commensurable periods of the periodic orbits.

PACS numbers: 03.65.Sq, 05.45.+b
\end{abstract}

\section{INTRODUCTION}

Quantum chaology has attracted the attention of the physics community after the discovery that the spectral correlations of classically chaotic systems are universal. They exhibit a strong level repulsion, which induces a non-trivial two-point correlation function $R(\varepsilon)$ of the density of states.

The spectral rigidity of a generic shaotic system was computed for the first time by Berry 3 . He expanded the density of states over peripdic orbits by making use of Gutzwiller's trace formulat. He estimated number of orbits of a given dength from the Hannay - Ozorio De Almeida sum rulel and computed the so-called diagonal part of the form-factor $K_{\text {diag }}(\tau)$. The form-factor $K(\tau)$ is a function of time, and is the Fourier transform of the two-point correlation function $R(\varepsilon)$.

The semiclassical theory of the form-factor distinguishes between three main time scales, see diagram Fig. 1(a). The contribution of the short periodic orbits is shown schematically as a sequence of $\delta$-peaks, and the relevant time scale is the period of the shortest periodic orbit $\tau_{p}$. This non-universal behavior of the form-factor prevails between $\tau=0$ and the ergodic time $\tau_{\text {erg }}$. Berry found that $K(\tau) \propto \tau$ for $\tau_{H} \gtrsim \tau \gtrsim \tau_{\text {erg }}$, and showed that this explains the level repulsion.

In the present work we are primarily interested in the semiclassical (periodic-orbit) theory of the form-factor for $\tau \gtrsim \tau_{H}$, where $\tau_{H}$ is the Heisenberg time. This is the third time scale. It is of quantum mechanical nature, because the Heisenberg time is proportional to the mean density of states. In Fig. 1 we have chosen units of time such that $\tau_{H}=1$. Due to the discrete nature of the density of states, the form-factor becomes constant for times much larger than $\tau_{H}$, see Fig. 1(a). The semiclassical theory for the form-factor for $\tau \gtrsim \tau_{H}$ has been analyzed recently by Bogomolny and Keating. They found the fingerprints of the short periodic orbits in the vicinity of the Heisenberg time as shown in Fig. 11(a) by $\delta$-peaks near $\tau=\tau_{H}$.

The most difficult problem addressed by Bogomolny and Keating was the computation of the off-diagonal part of the two-point correlation function $R_{\text {off }}(\varepsilon)$. Argaman et a 1 pointed out that it is possible to compute $R_{\text {off }}(\varepsilon)$ if the two-point statistics of the classical actions is known. Let us call the set of actions of all primitive periodic orbits (PPOs) the length spectrum. The density of states is related to the length spectrum by the Gutzwiller trace formulat. Therefore correlations of eigenenergies should be related to correlations of actions.

Our approach to the semiclassical evaluation of $B_{\text {off }}(\varepsilon)$ further develops the approach of Argaman et a $\mathbf{a}$ and their followers 10 but makes use of a different starting point. The first new element is the study of the spectral zeta function $\zeta(E)$, its autocorrelation function $C(\varepsilon)$, and its Fourier transform $K^{\zeta}(\tau)$.

The spectral zeta function $\zeta(E)$ is an important tool for an analytic or numeric computation of the energy levels of any quantum mechanical system. The zeros of this function are the eigenenergies of the Hamiltonian A unique definition of $\zeta(E)$ is to be given later. Voros 11 has proposed to compute this function by making use of a product over the periodic orbits. Berry and Keating 12 have expanded this product over the composite periodic orbits (CPOs), see precise formula below. The spectral zeta function is a smooth function that has no $\delta$-peaks like the density of states does. Therefore it is a good idea to characterize level statistics of chaotic systems by the $\zeta(E)$ autocorrelation function. This was done by Kette- 

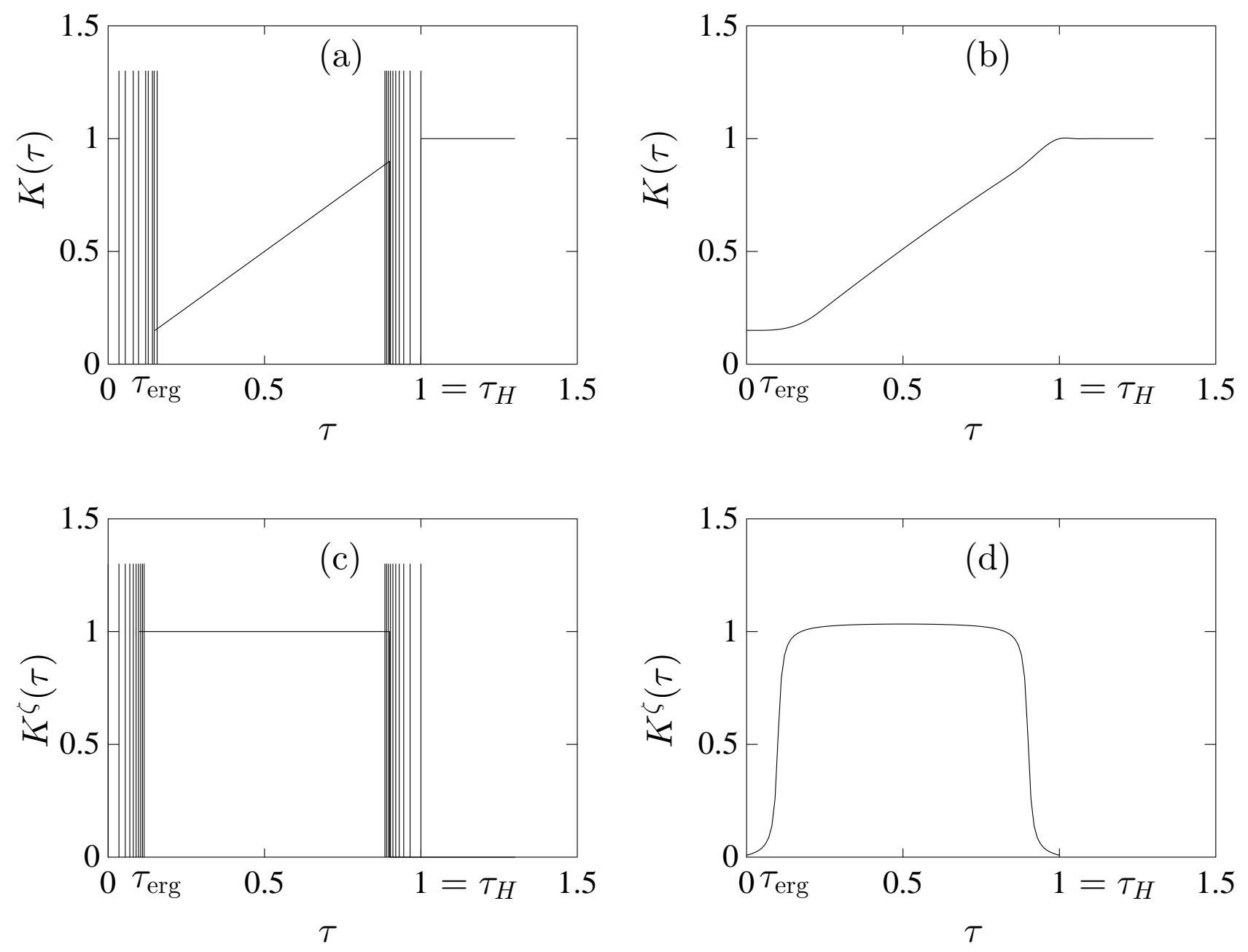

FIG. 1. Form-factors derived from correlation functions of density of states and spectral determinant. Diagrams (a), and (c) correspond to a small chaotic system, where one can observe fingerprints of short periodic orbits in the form-factors, shown as $\delta$-functional peaks. Diagrams (b) and (d) correspond to diffusive systems, which can be considered as huge complex chaotic systems. One can observe smoothing of the form-factors near $\tau=0$ and $\tau=\tau_{H}$. Particular shape of this smoothing contains system specific information like dimensionality and characteristics of disorder. 
mann, Klakow and Smilansky, who also computed this autocorrelation function $C(\varepsilon)$ for two and three dimensional Sinai billiards. 13

The diagram Fig. 11(c) schematically shows $K^{\zeta}(\tau)$. For systems with broken time reversal symmetry, as in Fig. 1, one can see the separation of the time scales very clearly. The short time behavior of $K^{\zeta}(\tau)$ is determined by the short CPOs, and it is represented by $\delta$-peaks. They become dense giving a constant behavior of $K^{\zeta}(\tau)$ for $\tau>\tau_{\text {erg }}$, see Ref. 13. It is known that the spectral zeta function has to satisfy a functional equation.14 This equation can be derived from the definition of $\zeta(E)$ and implies an exact mirror symmetry of $K^{\zeta}(\tau)$ around the half Heisenberg time: $K^{\zeta}\left(\tau_{H} / 2+\tau\right)=K^{\zeta}\left(\tau_{H} / 2-\tau\right)$. For this reason one has to observe the fingerprints of the short composite periodic orbits near $\tau_{H}$; they are shown as $\delta$-peaks near $\tau_{H}$ in Fig. 1 (c). Therefore, if the short time behavior of $K^{\zeta}(\tau)$ is known, then the behavior of $K^{\zeta}(\tau)$ near $\tau_{H}$ is also known.

Similar to the case of $R(\varepsilon)$, the periodic orbits expansion of $\zeta(E)$ allows one to separate $C(\varepsilon)$ and $K^{\zeta}(\tau)$ into diagonal and off-diagonal parts. Following Berry we can assume that the off-diagonal part of $K^{\zeta}(\tau)$ vanishes for $\tau<\tau_{H} / 2$. This assumption together with the mirror symmetry of $K^{\zeta}(\tau)$ implies an explicit connection between the diagonal and the off-diagonal parts of $K^{\zeta}(\tau)$. Physically, this relation has to be interpreted as a quantum-classical time scale separation.

Since $\zeta(E)$ is expressed in terms of CPOs by the Berry - Keating formula12, the autocorrelation function $C(\varepsilon)$ is related to correlations in the composite length spectrum, which is the set of all CPOs. In this way we introduce an analog of length correlations, which were used by Argaman et a to discuss the two-point spectral functions. One of the central ideas of the present work is to compute the correlations in the composite length spectrum by making use of the mirror symmetry of $K^{\zeta}(\tau)$. More over, the time scale separation provides an explicit dependence of $K^{\zeta}(\tau)$ on $\tau_{H}$. The two point correlation function of composite actions is just the Fourier transform of $K^{\zeta}(\tau)$ with respect to $1 / \hbar$ hidden in $\tau_{H}$. This idea is similar to one which has been suggested by Balian and Bloch.15

The length or the action of the given composite periodic orbit is simply the algebraic sum of the lengths or the actions of the PPOs forming this composite orbit. For this reason, action correlations of CPOs have to be related to action correlations of PPOs. In the present work we construct an integral relation between these correlation functions by making use of simple probabilistic arguments. This relation is generic and independent of the dimensionality of the system, however it might be dependent on the symmetry of the system. We perform calculations only for systems which does not have spatial symmetries, because we assume that the composite length spectrum is non-degenerate.

The statistical relation between correlations of CPOs and correlations of PPOs can be converted by the inverse Fourier transform and a special regularization procedure to a relation between the off-diagonal parts of $K(\tau)$ and $K^{\zeta}(\tau)$. Calculations show that the behavior of $K(\tau)$ near $\tau_{H}$, which is shown schematically in Fig. 1 (a), reproduces the behavior of $K^{\zeta}(\tau)$ near $\tau_{H}$, see Fig. 11(c).

The ideas of this paper are best understood by the following logical flow:

1. The off-diagonal part of $K(\tau)$ is the Fourier transform with respect to $1 / \hbar$ of the two-point statistics of actions of PPOs.

2. The two-point statistics of actions of PPOs can be computed from the two-point statistics of actions of CPOs.

3. The two-point statistics of actions of CPOs is determined by the off-diagonal part of $K^{\zeta}(\tau)$.

4. The diagonal and off-diagonal parts of $K^{\zeta}(\tau)$ are connected, because $\zeta(E)$ satisfies the functional equation.

5. For the systems with broken time reversal symmetry there is a clear separation between the classical scale (time of mixing) and the quantum mechanical scale (time of quantum recurrence). In this case we can compute the off-diagonal part of $K^{\zeta}(\tau)$ explicitly, if the diagonal part is known.

6. The diagonal parts of either $K(\tau)$ and $K^{\zeta}(\tau)$ or $R(\varepsilon)$ and $C(\varepsilon)$ have well known periodic orbit expansions, which can be evaluated poth numerically and by making use of sum rules 3,13

The two-point statistics of actions is a property of classical dynamics and it is independent of $\hbar$. Unfortunately, there are only a few examples of chaotic systems where a sufficient number of periodic orbits is known and the action correlations can be computed directly from the classical Hamiltonian.10 We circumvent this difficulty by computing action correlations from another source, namely from the diagqual part of $C(\varepsilon)$, which is also a purely classical object.13

The integral relation connecting the correlations of the PPOs and the CPOs can be applied directly to derive the well known correlations between prime numbers. As we show in Appendix B, the Hardy - Littlewood expression 16 can be reproduced in this framework. We emphasize this application as a very stringent test for our probabilistic method.

Large chaotic systems, for example billiards with a large number of scatterers or disordered systems, usually have $\tau_{\text {erg }} \gg \tau_{p}$, since $\tau_{\text {erg }}$ is the time of a diffusion through the system. The system specific features of $K(\tau)$ and $K^{\zeta}(\tau)$ are smooth, see Figs. 11(b) and $\mathbb{1}(\mathrm{d})$. The condition $\tau_{\text {erg }} \gg \tau_{p}$ allows us to express the diagonal part of $C(\varepsilon)$ as the Fredholm determinant of the diffusion propagator. In this way our expressions for $R(\varepsilon)$ reproduce precisely the field theory result of Andreev and Altshuler.17 
At this point it is appropriate to review the important work on the level statistics of disordered systems which had a very important impact on the development of the semiclassical theories presented here and elsewhere.110 Field theory was used by Efetov to compute the leve statistics of small metallic samples, see the review paper 18 . He considered the electron moving in a random potential of impurities and confined by sample boundaries. He assumed that the fluctuations of the fields are uniform across the sample and obtained universal results for the level statisfics.

Altshuler and Shklovskii 19 made use of perturbation theory and expressed the so-called perturbative part of $R(\varepsilon)$ in terms of the density-density correlation function, which is the propagator of the diffusion equation. Argaman et a 20 showed that the perturbative part of $R(\varepsilon)$ is nothing but the diagonal part of $R(\varepsilon)$ mentioned before.

The diagonal (or perturbative) part of $R(\varepsilon)$ is singular at small energies; the only way to remove this singularity is to compute the off-diagonal ( $\rho$ non-perturbative) part of $R(\varepsilon)$. Andreev and Altshuler 17 computed this term by making use of non-perturbative field theory and obtained the answer in terms of the same density-density correlation function. These authors repeated Efetov's calculations, but allowed the spatial fluctuations of the superfields.

The free energy functional in Efetov's theory describes the diffusion modes. These modes have to satisfy the diffusion equation. This theory was remarkably generalized by Muzykantskii and Khmelnitskii21. They obtained the free energy functional describing the eigenmodes of the kinetic equation. Such solutions to the kinetic equation have a meaning of the density-density correlation 22.

Muzykantskii and Khmelnitskii also suggested that the modes of the kinetic equation should be replaced by the modes of the Liouvillian operator if one goes from diff $\mu$ sive to chaotic systems. Agamet a 23 and Andreev et a 24 constructed field theory for chaotic systems by averaging over the energy and obtained the Liouvillian operator in the kinetic part of the free energy functional. Both types of field theory show that the spectral statistics of a chaotic system is described by the determinant of the Liouvillian operator.

The connection between the diagonal part of the correlation function and the determinant of the Liouvillian operator is almost trivial in the framework of periodic orbit theory. This connection is different from the field theory prediction due to the terms containing repetitions of the PPOs. The periodic orbit theory expression for the off-diagonal part of the cprrelation function obtained by Bogomolny and Keating is also different from the field theory result 24. Therefore, the derivation of the Andreev - Altshuler result in terms of the action correlations is interesting, and it also gives additional information about the autocorrelation function of the spectral determinant. The latter has been obtained by using random matrix theory 25.26 and random polynomial theory 27, as opposed to field theory.
All correlation functions employed in the present work are defined in Secs. II and III. In Sec. IV very simple probabilistic arguments will help us to build the integral equation connecting the correlations of CPOs with the correlations of PPOs. The behavior of form-factors near the Heisenberg time is considered in Sec. V. In the same section our results are compared with the universal random matrix theory predictions and the results of Refs. 17, 7. We discuss the physics of the action correlations in Sec. VI. We also summarize our results in Sec. VI.

\section{DEFINITIONS OF OBJECTS RELATED TO THE SPECTRAL CORRELATIONS.}

We start to build our theory for the specific example of the chaotic billiard. Let us also put the Aharonov-Bohm flux through the billiard in order to break the time reversal symmetry. Let us also assume that the system has no spatial symmetries. The classical motion of a charged particle in the billiard is finite and therefore one can associate two sets with this system. The first set, $\left\{l_{p}\right\}$, is formed by the lengths of all the PPOses, and each orbit is labeled by the index $p$. Each orbit makes $\mu_{p}$ windings around the Aharonov - Bohm flux. We distinguish the orbits with positive and negative winding numbers, i.e. the paths going clockwise and counterclockwise around the flux tube. Therefore, the length spectrum $\left\{l_{p}\right\}$ is degenerate, the lengths of the orbits with non-zero winding numbers appear twice.

The second set associated with the billiard is the quantum mechanical spectrum. It is formed by the wave vector magnitudes $\left\{k_{n}\right\}$. Each of them corresponds to the eigenvalue of the Hamiltonian $E_{n}=E\left(k_{n}\right)$ and $E(k)=\hbar^{2} k^{2} /(2 m)$, where $m$ is the mass of a particle.

The density of states is a sequence of $\delta$-peaks, and it is usually computed by making use of the Green function of the system:

$$
d(k) \equiv \sum_{n=1}^{\infty} \delta\left(k-k_{n}\right)=-\frac{1}{\pi} \operatorname{Im} \operatorname{Tr} \hat{G}^{r}(k) E^{\prime}(k) .
$$

Here $\hat{G}^{r}(k)$ is the retarded Green function of the system, and the prime means derivative with respect to $k$. The trace of $\hat{G}^{r}(k)$ can be represented as a sum over the peri odic orbits. Such an expansion is called a trace formula. The derivation of this expansion for a billiard can be found in the review Ref. 28. The result is

$\operatorname{Tr} \hat{G}^{r}(k) E^{\prime}(k)=-\sum_{p} \sum_{r=1}^{\infty} \frac{i l_{p} e^{i k r l_{p}-i \nu_{p} \pi / 2+i \phi \mu_{p}}}{e^{\lambda_{p} r l_{p} / 2}-e^{-\lambda_{p} r l_{p} / 2}}-i \pi \bar{d}(k)$,

where the index $p$ runs over the PPOs, the length of each orbit is $l_{p}$, the Lyapunov exponent of each orbit is $\lambda_{p} l_{p}$, and the Maslov index is $\nu_{p}$. We also broke the time reversal symmetry by adding the phase due to Aharonov- 


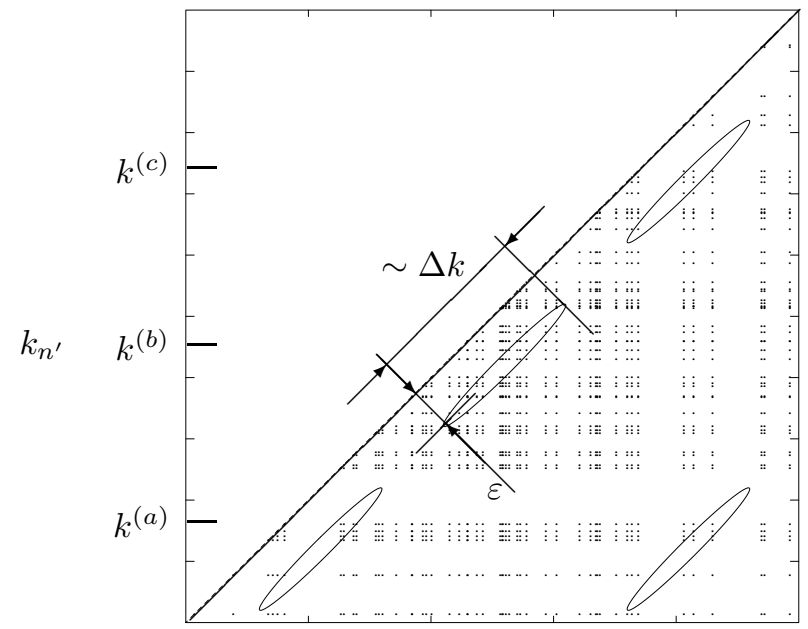

$k_{n}$

FIG. 2. Schematic diagram demonstrating how to compute the correlation function of energy levels. Each point represents pair of the energy levels $\left(k_{n}, k_{n^{\prime}}\right)$. The correlation function $R(\varepsilon, k)$ picks up the points inside one of the ellipses shown in the diagram. Three ellipses near the diagonal are drawn for $k$ equal to $k^{(a)}, k^{(b)}, k^{(c)}$, and $\varepsilon \ll \Delta k$. We draw the fourth ellipse for the case $\varepsilon \sim \Delta k$ and the choice of relevant $k$ becomes ambiguous.

Bohm flux $\phi$ multiplied by the winding number of the trajectory $\mu_{p}$. Lyapunov exponents in Eq. (1b) are defined per unit length of a trajectory and not per number of scatterings as in Ref. 28. This formula also contains the smooth part of the density of states $\bar{d}(k)$, which is proportional to the volume of the system. Equations (1a) and $(1 \mathrm{~b})$ show that the density of states contains an oscillating part. It is defined as

$$
d_{\mathrm{osc}}(k) \equiv d(k)-\bar{d}(k),
$$

and is proportional to the imaginary part of the first term on the right hand side of Eq. (1b).

The correlation function of the quantum mechanical spectrum and its Fourier transform have to be defined in a special way, because of the $\delta$-functional form of the density of states and the discrete form of the expansion Eq. (1b). We define the correlation function by making use of the averaging over $k$ with Gaussian weight

$$
\begin{aligned}
R(\varepsilon, k) & \equiv e^{-\Delta l^{2} \varepsilon^{2} / 2} \int_{-\infty}^{\infty} \frac{d q}{\sqrt{2 \pi} \Delta k} e^{-\frac{(q-k)^{2}}{2 \Delta k^{2}}} \\
& \times d_{\mathrm{osc}}\left(q+\frac{\varepsilon}{2}\right) d_{\mathrm{osc}}\left(q-\frac{\varepsilon}{2}\right)
\end{aligned}
$$

and this definition of the correlation function is valid for $\varepsilon \ll k$. We emphasize that $\varepsilon$ in our definition is the difference of the wave numbers and not of the energies as accepted.

The definition of the correlation function Eq. (2) contains the product of two $\delta$-functions from two densities of states and only one averaging. Practically, the $\delta$-function remaining after the integration in Eq. (2) has to be replaced by a smoothed function of the width smaller than the mean level spacing. Then, the definition Eq. (2) can be understood geometrically. The two-point correlation function is proportional to the difference between the number of the level pairs $\left(k_{n}, k_{n^{\prime}}\right)$ inside the region, which we showed schematically as an ellipse in Fig. 2, and the square of the mean density of states multiplied by the area of this region. The "length" of the ellipse is of the order of $\Delta k$ and the "width" has to be smaller than the mean level spacing.

We show three ellipses demonstrating the correlation function computed for $k$ equal to $k^{(a)}, k^{(b)}, k^{(c)}$, and $\varepsilon \ll$ $\Delta k$. It is important to note that $\Delta k$ has to be smaller than the characteristic scale of variation of $R(\varepsilon, k)$ as a function of $k$. We will see later that this scale is $\sim$ $\varepsilon \bar{d}^{\prime}(k)$, where the prime means derivative with respect to $k$. Therefore, the choice of the averaging interval length is limited by the inequality $\varepsilon \bar{d}^{\prime}(k) \Delta k \lesssim 1$.

The fourth ellipse in the diagram Fig. 2 is drawn in the area where $\varepsilon \sim \Delta k$. It is difficult to decide whether the value of $k$ in this case is $k^{(a)}$, or $k^{(b)}$, or maybe $k^{(c)}$. In order to make the correlation function well defined for all $\varepsilon$ we multiplied it by Gaussian prefactor $e^{-\Delta l^{2} \varepsilon^{2} / 2}$, see Eq. (2) where $\Delta l \Delta k \gtrsim 1$.

The domain of $\varepsilon$, where the correlation function is meaningful, is limited now by two competing conditions: $\varepsilon \Delta l \lesssim 1$ and $\varepsilon \lesssim 1 /\left(\Delta k \bar{d}^{\prime}\right)$. The latter inequality has to be implied by the former one, and therefore we obtain the important condition

$$
\frac{\Delta k}{\Delta l} \lesssim \frac{1}{\overline{d^{\prime}(k)}}
$$

The averaging has to be performed over a large number of the energy levels. This number is

$$
\Delta \mathcal{N} \sim \Delta k \bar{d}(k) \gg 1 .
$$

The inequalities Eqs. (3) and (41) are not very restrictive and most of the published experimental and numerical work, where the correlation function was computed, employed the averaging intervals of the width satisfying them.

Similar statistics can be defined for the length of the PPOs entering the right hand side of Eq. (1b). The length spectrum of the system has the density $\sum_{p} \delta(x-$ $\left.l_{p}\right)$, and the weighted mean density $\tilde{d}_{\text {ppo }}(x)$ is the sum over $n, \nu, \mu$ of

$$
\begin{aligned}
\bar{d}_{\mathrm{ppo}}(x, n, \nu, \mu) & =\sum_{p} e^{-\lambda_{p} l_{p}} \delta_{n n_{p}} \delta_{\nu \nu_{p}} \delta_{\mu \mu_{p}} \frac{e^{-\frac{\left(x-l_{p}\right)^{2}}{2 \Delta l^{2}}}}{\sqrt{2 \pi} \Delta l} \\
& \equiv \bar{d}_{\mathrm{ppo}}(X), \quad X=(x, n, \nu, \mu)
\end{aligned}
$$

where we have set the averaging interval to be precisely $\Delta l$, because we are going to build analytical relations between the statistics of energy levels and the statistics 


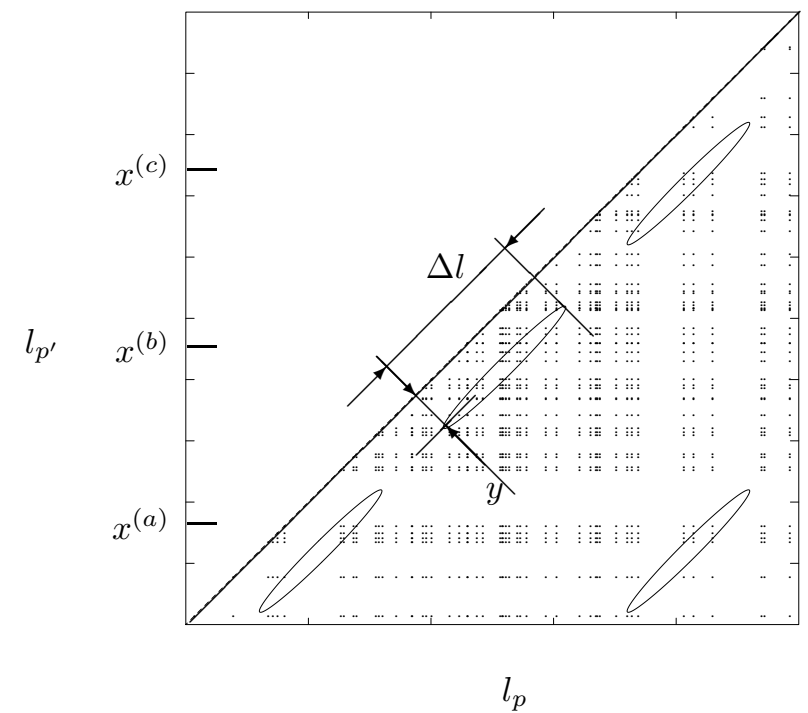

FIG. 3. Schematic diagram demonstrating how to compute the correlation function of the length spectrum. Each point represents the pair of the primitive periodic orbits lengths $\left(l_{p}, l_{p^{\prime}}\right)$. The correlation function $\tilde{R}(x, y)$ picks up the points inside one of the ellipses shown in the diagram. The three ellipses near the diagonal were drawn for $x$ equal to $x^{(a)}, x^{(b)}$, $x^{(c)}$, and $y \ll \Delta l$. The fourth ellipse was drawn for the case $y \sim \Delta l$ and the choice of relevant $x$ becomes ambiguous.

of periodic orbits. The mean density in Eq. (5) picks up the orbits $p$ with the defined value of the number of the wall reflections $n_{p}$. We keep this number in Eq. (5), because the action correlations were found between the orbits with the same $n_{p}$, see Ref. 10 .

The correlation function of the length spectrum is defined in a slightly different way from the correlation function of the density of states:

$$
\begin{aligned}
& \tilde{R}_{2}\left(x, n, \nu, \mu ; x^{\prime}, n, \nu^{\prime}, \mu^{\prime}\right)=\sum_{p} e^{-\frac{\lambda_{p} l_{p}}{2}} \delta_{n n_{p}} \delta_{\nu \nu_{p}} \delta_{\mu \mu_{p}} \\
& \times\left\{\sum_{p^{\prime}} e^{-\frac{\lambda_{p^{\prime}} l_{p^{\prime}}}{2}} \delta_{n n_{p}} \delta_{\nu^{\prime} \nu_{p^{\prime}}} \delta_{\mu^{\prime} \mu_{p^{\prime}}} \frac{e^{-\frac{\left(x-l_{p}\right)^{2}}{2 \Delta l^{2}}}}{\sqrt{2 \pi} \Delta l} \frac{e^{-\frac{\left(x^{\prime}-l_{p^{\prime}}\right)^{2}}{2 \Delta l^{2}}}}{\sqrt{2 \pi} \Delta l}\right. \\
& -\sum_{p^{\prime} \neq p} e^{-\frac{\lambda_{p^{\prime}} l_{p^{\prime}}}{2}} \delta_{n^{\prime} n_{p^{\prime}}} \delta_{\nu^{\prime}} \nu_{p^{\prime}} \delta_{\mu^{\prime} \mu_{p^{\prime}}} \delta\left(x-x^{\prime}-l_{p}+l_{p^{\prime}}\right) \\
& \left.\times \frac{e^{-\frac{1}{2 \Delta l^{2}}\left(\frac{x+x^{\prime}}{2}-\frac{l_{p}+l_{p^{\prime}}}{2}\right)^{2}}}{\sqrt{2 \pi} \Delta l}\right\} e^{-\Delta k^{2}\left(x-x^{\prime}\right)^{2} / 2} \\
& \equiv \tilde{R}_{2}\left(X ; X^{\prime}\right)
\end{aligned}
$$

where the first term in the braces is the product of the mean densities of PPOs and the second term is the probability of finding two PPOs having the length difference $y$. Both terms are weighted by stability factors which compensate the exponential proliferation of the PPOs.

The key assumption of the present theory is that the orbits with different Maslov indexes or different winding numbers or different number of scatterings do not con- tribute to the correlation function of the length spectrum, which we should define as

$$
\tilde{R}(x, y)=\sum_{n, \nu, \mu} \tilde{R}_{2}\left(x+\frac{y}{2}, n, \nu, \mu ; x-\frac{y}{2}, n, \nu, \mu\right) .
$$

Therefore we assume that $\tilde{R}_{2}\left(X ; X^{\prime}\right)$ decay very fast with $\left|n-n^{\prime}\right|,\left|\nu-\nu^{\prime}\right|$, and $\left|\mu-\mu^{\prime}\right|$. The correlation function $\tilde{R}_{2}\left(X ; X^{\prime}\right)$ is meaningful for $\left|x-x^{\prime}\right| \lesssim \Delta l$ and this is taken into account by the factor $e^{-\Delta k^{2}\left(x-x^{\prime}\right)^{2} / 2}$ in Eq. (6a), since $1 / \Delta k \lesssim \Delta l$ as we will show later.

The correlation function defined by Eq. (6b) has a normalization condition which is an extremely useful tool for verifying results. We have from Eqs. (6)

$$
\begin{aligned}
\sum_{n^{\prime} \nu^{\prime} \mu^{\prime}} \int d x^{\prime} \tilde{R}_{2}\left(X ; X^{\prime}\right) & =\bar{d}_{\mathrm{ppo}}(X) \\
\int d y \tilde{R}(x, y) & =\tilde{d}_{\mathrm{ppo}}(x)
\end{aligned}
$$

which are valid under the condition $\Delta l \ll x$. In order to derive this relation we have replaced $\sum_{p} \sum_{p^{\prime} \neq p}$ in Eq. (6a) by two terms $\sum_{p p^{\prime}}-\sum_{p} \delta_{p p^{\prime}}$. The second term gives the mean density on the right hand side of Eqs. (7). The first term is a product of the mean densities and cancels another term of the type $\sum_{p p^{\prime}}$ in Eq. (6a).

The correlation functions defined by Eqs. (6) have the graphical representation in Fig. 3 quite similar to that in Fig. 2. In the case of Fig. 3 we are interested in the number of PPO pairs whose mean length is near $x$ and difference of lengths is near $y$. Correlation function counts the number of PPO pairs inside one of the ellipses shown in Fig. 3. The "length" of the ellipses is determined by the width of the Gaussians in Eq. (6a) and to be $\sim \Delta l$. The "width" of the ellipses does not appear explicitly in Eq. (6a), meaning that $\delta\left(y-l_{p}+l_{p^{\prime}}\right)$ has to be smoothed to the order of $1 / \tilde{d}_{\mathrm{ppo}}(x)$.

The length of the averaging interval, $\Delta l$, has to be as large as possible, but smaller than the characteristic scale of the variation of $\tilde{R}(x, y)$ as a function of $x$ for constant $y$. This scale can be estimated from the results of Ref. 8 and it is $\bar{d}^{\prime}(k) / y$ taken for $k=k_{x}$, where $k_{x}$ is the inverse density of states:

$$
x=2 \pi \bar{d}\left(k_{x}\right),
$$

which is a classical quantity. The choice of the averaging interval is, therefore, limited by the inequality $\Delta l \lesssim \bar{d}^{\prime}(k) / y$. This inequality has to be fulfilled in the interval of $y$, where the correlation function Eq. (6) is meaningful, i.e. for $0<y \lesssim 1 / \Delta k$. Therefore, the choice of the averaging window width is limited by the condition analogous to Eq. (4)

$$
\frac{\Delta l}{\Delta k} \lesssim \bar{d}^{\prime}\left(k_{x}\right) .
$$

This inequality holds regardless of Eq. (4) because one can study the statistics of energy levels independently 
from the statistics of periodic orbits. From this point of view the inequality Eq. (9) is not very restrictive either.

The statistical properties of the PPOs length spectrum are ultimately connected with the statistical properties of the quantum mechanical spectrum 8 . We obtain from Eqs. (11) the periodic orbit expansion of the spectral correlations form-factor

$$
\begin{aligned}
K(x, k) & \equiv \int_{-\infty}^{\infty} \frac{d \varepsilon}{2 \pi} e^{-i \varepsilon x} R(\varepsilon, k)=K_{\mathrm{diag}}(x)+K_{\mathrm{off}}(x, k) \\
K_{\mathrm{diag}}(x) & =\frac{1}{4 \pi^{2}} \sum_{p r} \frac{l_{p}^{2}}{\left|e^{\frac{\lambda_{p} r l_{p}}{2}}+e^{-\frac{\lambda_{p} r l_{p}}{2}}\right|^{2}} \frac{e^{-\frac{1}{2 \Delta l^{2}}\left(|x|-l_{p} r\right)^{2}}}{\sqrt{2 \pi} \Delta l} \\
K_{\mathrm{off}}(x, k) & =\sum_{p} \sum_{p^{\prime} \neq p} \frac{l_{p} l_{p^{\prime}} e^{-\frac{\lambda_{p} l_{p}+\lambda_{p^{\prime}} l_{p^{\prime}}}{2}}}{4 \pi^{2}} \frac{e^{-\frac{1}{2 \Delta l^{2}}\left(|x|-\frac{l_{p}+l_{p^{\prime}}}{2}\right)^{2}}}{\sqrt{2 \pi} \Delta l} \\
& \times e^{i k\left(l_{p}-l_{p^{\prime}}\right)-\Delta k^{2}\left(l_{p}-l_{p^{\prime}}\right)^{2} / 2} \\
& \times e^{i\left(\nu_{p}-\nu_{p^{\prime}}\right) \pi / 2+i \phi\left(\mu_{p}-\mu_{p^{\prime}}\right)} \quad(10 \mathrm{c}) \\
& =-\frac{1}{4 \pi^{2}} x^{2} \int_{-\infty}^{\infty} d y e^{i k y} \tilde{R}(|x|, y)
\end{aligned}
$$

which is valid for $k \gg \Delta k$. Under this condition we have neglected the Fourier transform of the first term in the braces on the left hand side of Eq. (6a), because it contains the factor $e^{-k^{2} /\left(2 \Delta k^{2}\right)}$.

Equation (10d) is justified for $x$ such that

$$
\lambda|x| \gg 1
$$

where $\lambda$ is the mean Lyapunov exponent defined per unit length of the trajectory. This condition allows us to expand the denominators in Eq. (11b) and to neglect the summation over the repetition index $r$. We also assumed that the magnetic flux $\phi$ is so large that $\phi \Delta \mu \gg 1$, where $\Delta \mu$ is the characteristic scale of $\mu-\mu^{\prime}$-dependence of $\tilde{R}_{2}\left(X ; X^{\prime}\right)$.

The key question of the theory is how to perform the summation over the periodic orbits if the length spectrum is unknown. This question was resolved $\mathrm{B}$ with the help of the sum rule b $^{5}$ valid for ergodic systems. One can deduce the density of PPOs from this sum rule:

$$
\tilde{d}_{\mathrm{ppo}}(x) \sim \frac{1}{x}
$$

This expression is valid asymptotically for $x$ larger than the ergodic length ( $\tau_{\text {erg }}$ times velocity) and it gives $K_{\text {diag }}(x) \propto x$.

The definition of the form-factor, Eqs. (10), is not complete, because we did not specify yet how to choose the sizes of the averaging windows $\Delta k$ and $\Delta l$. Common wisdom was that Eqs. (10) would survive if they do not break the conditions

$$
\Delta l e^{\lambda x} / x \gg 1, \quad \Delta k \bar{d}(k) \gg 1 .
$$

We want to define the form-factor in such a way that it will carry information about the correlations of energy levels and about the correlations of periodic orbits. Therefore, we require $\Delta k$ and $\Delta l$ to satisfy Eqs. (4) and (9) i.e.

$$
\bar{d}^{\prime}(k) \lesssim \frac{\Delta l}{\Delta k} \lesssim \bar{d}^{\prime}\left(k_{x}\right)
$$

Near the Heisenberg length $x \sim 2 \pi \bar{d}(k)$, we have $k_{x}=k$ and two inequalities in Eq. (14) give us $\Delta l / \Delta k \sim \bar{d}^{\prime}(k)$.

\section{DEFINITIONS OF OBJECTS RELATED TO THE SPECTRAL ZETA FUNCTION.}

One can construct an infinitely large number of complex analytic functions of $k$ having zeros only at $k=k_{n}$. Each such function can be considered as a spectral determinant. We are going to define the spectral determinant in terms of the retarded Green function $\hat{G}^{r}(k)$. The trace of this operator is an analytic function and we have

$$
\zeta(k) \equiv e^{\int_{0}^{k} d q\left\{\operatorname{Tr} \hat{G}^{r}(q) E^{\prime}(q)+i \pi \bar{d}(q)\right\}} .
$$

This definition implies the functional equation for the spectral zeta function

$$
\zeta(k)=e^{2 \pi i \int_{0}^{k} d q \bar{d}(q)} \zeta^{*}(k),
$$

where the asterisk stands for the complex conjugation. The expression in braces in Eq. (15) can be written as the series expansion over the periodic orbits Eq. ( $1 \mathrm{~b})$. The exponential function of this series was computed by Voros 11 and the result is

$$
\zeta(k)=\prod_{p} \prod_{r=0}^{\infty}\left(1-e^{i\left(k l_{p}-\nu_{p} \pi / 2+\phi \mu_{p}\right)} e^{-(r+1 / 2) \lambda_{p} l_{p}}\right) .
$$

This expression is taken from Eq. (6.21) of Ref. 28, and $\lambda_{p}$ here is the Lyapunov exponent per unit length. Here we added the phase due to the Aharonov - Bohm flux.

The autocorrelation function of the spectral zeta is defined similarly to the correlation function of the density of states:

$$
\begin{aligned}
C(\varepsilon, k) & \equiv e^{-\Delta l^{2} \varepsilon^{2} / 2} \int_{-\infty}^{\infty} \frac{d q}{\sqrt{2 \pi} \Delta k} e^{-\frac{(q-k)^{2}}{2 \Delta k^{2}}} \\
& \times \zeta\left(q+\frac{\varepsilon}{2}\right) \zeta^{*}\left(q-\frac{\varepsilon}{2}\right)
\end{aligned}
$$

Equation. (16) is also valid for the correlation function

$$
C(\varepsilon, k)=e^{2 \pi i \bar{d}(k) \varepsilon} C(-\varepsilon, k),
$$

where we expanded the integral in the exponent in Eq. (16) under the condition $\varepsilon \ll k$, and also used the inequality Eq. (4). 
As usual, we put the prefactor in Eq. (18), which will be translated into the averaging over the lengths of the CPOs. The CPOs appear from the evaluation of the product in Eq. (17) and we define them as all possible sets of the PPOs taken without repetitions:

$$
c=\{p\} \text { and } l_{c} \equiv \sum_{p \in c} l_{p}
$$

The CPOs have the composite Lyapunov exponents $\lambda_{c} \equiv \frac{1}{l_{c}} \sum_{p \in c} \lambda_{p} l_{p}$, the composite Maslov indexes $\nu_{c} \equiv$ $\sum_{p \in c} \nu_{p}$, the composite number of wall reflections $n_{c} \equiv$ $\sum_{p \in c} n_{p}$, the number of the primitive components $m_{c} \equiv$ $\sum_{p \in c} 1$, and the composite winding numbers $\mu_{c} \equiv$ $\sum_{p \in c} \mu_{p}$. This definition of the CPOs leads to the high degeneracy of the composite length spectrum $\left\{l_{c}\right\}$.

The composite length spectrum has the density $\sum_{c} \delta\left(x-l_{c}\right)$ and the weighted mean density $\tilde{d}_{\text {cpo }}(x)$ is the sum over $n, \nu, \mu$ of

$$
\begin{aligned}
\bar{d}_{\mathrm{cpo}}(x, n, \nu, \mu) & =\sum_{c} e^{-\lambda_{c} l_{c}} \delta_{n n_{p}} \delta_{\nu \nu_{p}} \delta_{\mu \mu_{p}} \frac{e^{-\frac{\left(x-l_{c}\right)^{2}}{2 \Delta l^{2}}}}{\sqrt{2 \pi} \Delta l} \\
& \equiv \bar{d}_{\mathrm{cpo}}(X)
\end{aligned}
$$

The correlation function of the composite length spectrum is defined similarly to Eq. (6)

$$
\begin{aligned}
& \tilde{C}_{2}\left(x, n, \nu, \mu ; x^{\prime}, n, \nu^{\prime}, \mu^{\prime}\right)=\sum_{c} e^{-\frac{\lambda_{c} l_{c}}{2}} \delta_{n n_{c}} \delta_{\nu \nu_{c}} \delta_{\mu \mu_{c}} \\
& \times\left\{\sum_{c^{\prime}} e^{-\frac{\lambda_{c^{\prime} l_{c^{\prime}}}}{2}} \delta_{n n_{c}} \delta_{\nu^{\prime} \nu_{c^{\prime}}} \delta_{\mu^{\prime} \mu_{c^{\prime}}} \frac{e^{-\frac{\left(x-l_{c}\right)^{2}}{2 \Delta l^{2}}} \frac{e^{-\frac{\left(x^{\prime}-l_{c^{\prime}}\right)^{2}}{2 \Delta l^{2}}}}{\sqrt{2 \pi} \Delta l} \frac{{ }^{2 \pi} \Delta l}{}}{-\sum_{c^{\prime} \neq c} e^{-\frac{\lambda_{c^{\prime}} l_{c^{\prime}}}{2}} \delta_{n^{\prime} n_{c^{\prime}}} \delta_{\nu^{\prime} \nu_{c^{\prime}}} \delta_{\mu^{\prime} \mu_{c^{\prime}}} \delta\left(x-x^{\prime}-l_{c}+l_{c^{\prime}}\right)}\right. \\
& \left.\times \frac{e^{-\frac{1}{2 \Delta l^{2}}\left(\frac{x+x^{\prime}}{2}-\frac{l_{c}+l_{c^{\prime}}}{2}\right)^{2}}}{\sqrt{2 \pi} \Delta l}\right\} e^{-\Delta k^{2}\left(x-x^{\prime}\right)^{2} / 2} \\
& \equiv \tilde{C}_{2}\left(X ; X^{\prime}\right), \\
& \tilde{C}(x, y)=\sum_{n, \nu, \mu} \tilde{C}_{2}\left(x+\frac{y}{2}, n, \nu, \mu ; x-\frac{y}{2}, n, \nu, \mu\right)
\end{aligned}
$$

We will assume in what follows that $\tilde{C}_{2}\left(X ; X^{\prime}\right)$ falls off rapidly with $\left|n-n^{\prime}\right|,\left|\nu-\nu^{\prime}\right|$ and $\left|\mu-\mu^{\prime}\right|$. The correlation function $\tilde{C}(x, y)$ is meaningful only for $y \lesssim \Delta l$, and this is taken into account by the Gaussian prefactor. The normalization ofthe correlation function can be deduced from Eqs. 21 and (22)

$$
\begin{aligned}
\sum_{n^{\prime} \nu^{\prime} \mu^{\prime}} \int_{0}^{\infty} d x^{\prime} \tilde{C}_{2}\left(X ; X^{\prime}\right) & =\bar{d}_{\mathrm{cpo}}(X), \\
\int_{-\infty}^{\infty} d y \tilde{C}(x, y) & =\tilde{d}_{\mathrm{cpo}}(x),
\end{aligned}
$$

and it is valid under the condition $\Delta l \ll x$. The derivation is similar to the derivation of Eq. (耳).
The statistical properties of the CPOs length spectrum are connected with the statistical properties of the spectral zeta function. We can see that by expanding the Fourier transform of the correlation function Eq. (18) over the CPOs

$$
\begin{aligned}
K^{\zeta}(x, k) & \equiv \int_{-\infty}^{\infty} \frac{d \varepsilon}{2 \pi} e^{-i \varepsilon x} C(\varepsilon, k)=K_{\mathrm{diag}}^{\zeta}(x)+K_{\mathrm{off}}^{\zeta}(x, k) \\
K_{\mathrm{diag}}^{\zeta}(x) & =\sum_{c} \sum_{r_{p}, p \in c}\left\{\prod_{p \in c} \frac{e^{-\lambda_{p} l_{p} r_{p}^{2}}}{\prod_{j=1}^{r_{p}}\left(1-e^{-\lambda_{p} l_{p} j}\right)^{2}}\right\} \\
& \times \frac{1}{\sqrt{2 \pi} \Delta l^{2}} e^{-\left(x-\sum_{p \in c} l_{p} r_{p}\right)^{2} /\left(2 \Delta l^{2}\right)} \\
& \approx \tilde{d}_{\mathrm{cpo}}(x) \\
K_{\mathrm{off}}^{\zeta}(x, k) & \approx \sum_{c} \sum_{c^{\prime} \neq c} e^{-\frac{\lambda_{c} l_{c}+\lambda_{c^{\prime}} l_{c^{\prime}}}{2}} \frac{e^{-\frac{1}{2 \Delta l^{2}}\left(|x|-\frac{l_{c}+l_{c^{\prime}}}{2}\right)^{2}}}{\sqrt{2 \pi} \Delta l^{2}} \\
& \times e^{i k\left(l_{c}-l_{c^{\prime}}\right)-\Delta k^{2}\left(l_{c}-l_{c^{\prime}}\right)^{2} / 2} e^{i \pi\left(m_{c}-m_{c}^{\prime}\right)} \\
& \times e^{i\left(\nu_{c}-\nu_{c^{\prime}}\right) \pi / 2} e^{i \phi\left(\mu_{c}-\mu_{c^{\prime}}\right)} \\
& =-\int_{-\infty}^{\infty} d y e^{i k y} \tilde{C}(x, y),
\end{aligned}
$$

where the repetition indexes $r_{p}$ in Eq. (25b) run from 1 to $\infty$. Equations (25d), (25d) and (25d) are valid under the condition Eq. (11). This condition allows us to keep only the factors with $r=0$ in Eq. (17), and therefore our definition of the CPOs Eq. (20) does not contain the repetitions of the PPOs. We also assumed that the magnetic flux $\phi$ is so large that $\phi \Delta \mu \gg 1$, where $\Delta \mu$ is the characteristic scale of the $\mu-\mu^{\prime}$-dependence of $\tilde{C}_{2}\left(X ; X^{\prime}\right)$. Equation (25a) defines the form-factor of the autocorrelation function of the spectral zeta. It is zero for negative $x$, whereas $K(x)$ depends on $|x|$.

The averaged density of the CPOs length spectrum was computed 29 for a family of strongly chaotic systems and its asymptotic form in the general case is

$$
\tilde{d}_{\mathrm{cpo}}(x) \sim \gamma,
$$

where $\gamma$ is the normalization constant, and $\gamma^{-1}$ is of the order of the billiard size. This density is sufficient for the computation of the diagonal part of the form-factor Eq. 25c) but the non-diagonal part can be computed only if the correlations in the CPOs are known.

\section{PROBABILISTIC THEORY OF THE LENGTH SPECTRUM CORRELATIONS.}

The length spectrum of PPOs is a part of the length spectrum of the CPOs. Therefore we can ask what is the probability to find a PPO in some interval of the composite length spectrum. Let $\mathbf{P}$ be the set of all PPOs and let $\mathbf{C}$ be the set of all CPOs. Then $\mathbf{P} \subset \mathbf{C}$. It 
may happen that $c \in \mathbf{C}$ consists of only one PPO and therefore $c \in \mathbf{P}$. We define the probability of this event

$$
P(c) \equiv\langle c \in \mathbf{P}\rangle_{l_{c}}=\frac{\bar{d}_{\mathrm{ppo}}\left(X_{c}\right)}{\bar{d}_{\mathrm{cpo}}\left(X_{c}\right)} .
$$

It is a function of $X_{c}$ where $X_{c}=\left(l_{c}, n_{c}, \nu_{c}, \mu_{c}\right)$. The probability $P(c)$ counts CPOs, which are actually PPOs, having defined values of $n_{c}, \nu_{c}, \mu_{c}$ and lengths which lie in the small interval near $l_{c}$. In Eq. (27) we regard the statement $c \in \mathbf{P}$ as a boolean function, which is equal to one if $c \in \mathbf{P}$ and equal to zero if $c \notin \mathbf{P}$.

We can also count the number of pairs of the CPOs, $c, c^{\prime} \in \mathbf{C}$ with the fixed length difference $l_{c}-l_{c^{\prime}}$, such that both of them are actually PPOs, $c, c^{\prime} \in \mathbf{P}$. The probability of finding such a pair is defined as

$$
\begin{aligned}
P\left(c, c^{\prime}\right) & \equiv\left\langle c \in \mathbf{P} \cap c^{\prime} \in \mathbf{P}\right\rangle_{\frac{l_{c}+l_{c^{\prime}}}{2}}=P(c) P\left(c^{\prime}\right) \\
& -e^{-\frac{\lambda_{c} l_{c}+\lambda_{c^{\prime}} l_{c^{\prime}}}{2}} \frac{\tilde{R}_{2}\left(X_{c} ; X_{c^{\prime}}\right)}{\bar{d}_{\mathrm{cpo}}\left(X_{c}\right) \bar{d}_{\mathrm{cpo}}\left(X_{c^{\prime}}\right)} .
\end{aligned}
$$

This equation defines the averaging over $\frac{l_{c}+l_{c^{\prime}}}{2}$. The fluctuations of the Lyapunov exponent are ignored here for two reasons: First, it was found numerically 10 that the fluctuations of the stability amplitudes do not affect the correlation function; second, our result Eq. (42) is independent of these fluctuations, because $P(c)$ is smooth function of $X_{c}$.

The probability that CPO $c$ contains another CPO $c^{\prime}$ can be defined by averaging over the length of $c$

$$
P\left(c^{\prime} \in c\right) \equiv\left\langle c^{\prime} \in c\right\rangle_{l_{c}}=e^{-\lambda_{c^{\prime}} l_{c^{\prime}}} \frac{\bar{d}_{\mathrm{cpo}}\left(X_{c}-X_{c^{\prime}}\right)}{\bar{d}_{\mathrm{cpo}}\left(X_{c}\right)}
$$

This probability is related to the probability $P(c)$ in Eq. (27) since

$$
c \in \mathbf{P} \Leftrightarrow \bigcap_{p \in \mathbf{P}, l_{p}<l_{c}^{*}} p \notin c,
$$

where $l_{c}^{*}$ is an arbitrary length such that $l_{c} / 2 \geq l_{c}^{*}<l_{c}$. Averaging over $l_{c}$ gives

$$
\begin{aligned}
P(c) & =\prod_{p, l_{p}<l_{c}^{*}}[1-P(p \in c)] \\
& +\sum_{c^{\prime}}(-1)^{m_{c^{\prime}}}\left[P\left(c^{\prime} \in c\right)-\prod_{p \in c^{\prime}} P(p \in c)\right],
\end{aligned}
$$

where the composite orbits $c^{\prime}$ are such that $l_{c^{\prime}} \geq l_{c}^{*}$ and $\forall p \in c^{\prime} \quad l_{p}<l_{c}^{*}$. We assume here that $P\left(c^{\prime} \in c\right)=$ $\prod_{p \in c^{\prime}} P(p \in c)$ unless $l_{c^{\prime}}$ is larger than $l_{c}^{*}$. One can choose $l_{c}^{*}$ by minimizing the absolute value of the second term on the right hand side of Eq. (31).

The main purpose of this section is to construct an equation analogous to Eq. (31) for the joint probability $P\left(c, c^{\prime}\right)$. Equation (30) for pair of CPOs reads

$$
c \in P \cap c^{\prime} \in P \Leftrightarrow \bigcap_{p \in \mathbf{P}, l_{p}<l_{c}^{*}} p \notin c \cap p \notin c^{\prime} .
$$

This statement can be converted to a Boolean expression and averaged over $\left(l_{c}+l_{c^{\prime}}\right) / 2$. That is

$$
\begin{aligned}
P\left(c, c^{\prime}\right)=\left\langle\prod _ { p , l _ { p } < l _ { c } ^ { * } } \left[ 1-p \in c-p \in c^{\prime}\right.\right. & \\
& \left.\left.+p \in c \cap p \in c^{\prime}\right]\right\rangle_{\frac{l_{c}+l_{c^{\prime}}}{2}}
\end{aligned}
$$

Let CPO $c^{\prime \prime}$ consist only of the PPOs which are shorter than $l_{c}^{*}$. We can introduce the sum over such orbits into Eq. (33)

$$
\begin{aligned}
& P\left(c, c^{\prime}\right)=\left\langle\sum_{c^{\prime \prime}} \prod_{p \notin c^{\prime \prime}, l_{p}<l_{c}^{*}}\left[1-p \in c-p \in c^{\prime}\right]\right. \\
& \left.\times \prod_{p \in c^{\prime \prime}}\left[p \in c \cap p \in c^{\prime}\right]+\prod_{l_{p}<l_{c}^{*}}\left[1-p \in c-p \in c^{\prime}\right]\right\rangle \\
& \approx \sum_{c^{\prime \prime}} \prod_{p \notin c^{\prime \prime}, l_{p}<l_{c}^{*}}\left[1-P(p \in c)-P\left(p \in c^{\prime}\right)\right] \\
& \times P\left(c^{\prime \prime} \in c \cap c^{\prime \prime} \in c^{\prime}\right)+\prod_{l_{p}<l_{c}^{*}}\left[1-P(p \in c)-P\left(p \in c^{\prime}\right)\right],
\end{aligned}
$$

where the probability of having a "common divisor" is given by

$$
P\left(c^{\prime \prime} \in c \cap c^{\prime \prime} \in c^{\prime}\right) \equiv\left\langle c^{\prime \prime} \in c \cap c^{\prime \prime} \in c^{\prime}\right\rangle_{\frac{l_{c}+l_{c^{\prime}}}{2}} .
$$

We assumed that for $p \neq p^{\prime}$

$$
\left\langle p \in c \cap p^{\prime} \in c^{\prime}\right\rangle_{\frac{l_{c}+l_{c^{\prime}}}{2}} \approx\langle p \in c\rangle_{l_{c}}\left\langle p^{\prime} \in c^{\prime}\right\rangle_{l_{c^{\prime}}}
$$

and also we made the same assumptions as in the derivation of Eq. (31). Particularly we neglected correction terms in Eq. (35), which are sums over $c^{\prime \prime}, l_{c^{\prime \prime}} \geq l_{c}^{*}$. Such correction terms were important in Eq. (31), but they can be neglected in Eq. (35) because the greatest contribution to the correlation of PPOs is given by the short CPOs $c^{\prime \prime}$.

It is convenient to rewrite Eq. (35) as a relation between the correlation function of PPOs and CPOs having a "common divisor"

$$
\begin{aligned}
& P(c) P\left(c^{\prime}\right)-P\left(c, c^{\prime}\right) \\
& \approx \sum_{c^{\prime \prime}}\left\{\left[P\left(c^{\prime \prime} \in c\right) P\left(c^{\prime \prime} \in c^{\prime}\right)-P\left(c^{\prime \prime} \in c \cap c^{\prime \prime} \in c^{\prime}\right)\right]\right. \\
& \left.\quad \times \prod_{p \notin c^{\prime \prime}, l_{p}<l_{c}^{*}}\left[1-P(p \in c)-P\left(p \in c^{\prime}\right)\right]\right\} .
\end{aligned}
$$


It is essential for the derivation of Eq. (38) that the composite orbit $c^{\prime \prime}$ does not contain repetitions of the primitive orbits. This agrees with our definition of composite orbits Eq. (20). In general one can allow repetition of the primitive orbits in the definition of the composite orbit. In this case Eq. 38 is also correct, but the sum must be taken over the composite orbits which does not contain repetitions of the primitive orbits.

The derivation of Eq. (38) contains a number of approximations. Therefore, one would like to verify that this equation preserves the normalization of the correlation functions. This verification appears in Appendix A.

In certain cases, any given composite orbit contains a small number of PPOs and therefore we can make a further approximation in Eq. (38)

$$
\begin{aligned}
& \prod_{p \notin c^{\prime \prime}, l_{p}<l_{c}^{*}}\left[1-P(p \in c)-P\left(p \in c^{\prime}\right)\right] \\
\approx & \prod_{l_{p}<l_{c}^{*}}[1-P(p \in c)]\left[1-P\left(p \in c^{\prime}\right)\right] .
\end{aligned}
$$

The condition of a small number of "divisors"

$$
P(p \in c) \propto e^{-\lambda_{p} l_{p}} \ll 1
$$

is not enough to justify making this approximation. It might happen that the error becomes larger when the sum over $c^{\prime \prime}$ in Eq. (38) is computed. One can check that this error is of the same order as the contribution of the repetitions of PPOs which has been neglected.

From Eqs. (38) and (39) by making use of Eq. (31) we obtain

$$
\begin{aligned}
& P(c) P\left(c^{\prime}\right)-P\left(c, c^{\prime}\right) \approx P(c) P\left(c^{\prime}\right) \\
& \times \sum_{c^{\prime \prime}}\left[P\left(c^{\prime \prime} \in c\right) P\left(c^{\prime \prime} \in c^{\prime}\right)-P\left(c^{\prime \prime} \in c \cap c^{\prime \prime} \in c^{\prime}\right)\right] .
\end{aligned}
$$

The right hand side of Eq. (38) or Eq. (40) contains the non-trivial probability of finding a "common divisor" $P\left(c^{\prime \prime} \in c \cap c^{\prime \prime} \in c^{\prime}\right)$. It is difficult to find a "common divisor" if the lengths of $c$ and $c^{\prime}$ are close to each other. We can compute this probability by noting that in order to find a "common divisor" of length $l_{c^{\prime}}$ one has to find two CPOs $c^{i i i}$ and $c^{i v}$ of length $l_{c^{i i i}}=l_{c}-l_{c^{\prime \prime}}$ and $l_{c^{i v}}=l_{c^{\prime}}-l_{c^{\prime \prime}}$. The same is true for other components of $X_{c}$ characterizing each CPO. The probability of finding a pair of composite orbits of specific lengths is given by the two-point correlation function of the composite length spectrum. Therefore, we substitute

$$
\begin{aligned}
& P\left(c^{\prime \prime} \in c \cap c^{\prime \prime} \in c^{\prime}\right)=P\left(c^{\prime \prime} \in c\right) P\left(c^{\prime \prime} \in c^{\prime}\right) \\
& -e^{-\frac{\lambda_{c} l_{c}+\lambda_{c^{\prime}} l_{c^{\prime}}}{2}-\lambda_{c^{\prime \prime}} l_{c^{\prime \prime}}} \frac{\tilde{C}_{2}\left(X_{c}-X_{c^{\prime \prime}}, X_{c^{\prime}}-X_{c^{\prime \prime}}\right)}{\bar{d}_{\mathrm{cpo}}\left(X_{c}\right) \bar{d}_{\mathrm{cpo}}\left(X_{c^{\prime}}\right)} .
\end{aligned}
$$

into Eq. (40) and arrive at

$$
\begin{aligned}
& \tilde{R}_{2}\left(X_{c}, X_{c^{\prime}}\right) \approx P(c) P\left(c^{\prime}\right) \\
& \times \sum_{c^{\prime \prime}} e^{-\lambda_{c^{\prime \prime}} l_{c^{\prime \prime}}} \tilde{C}_{2}\left(X_{c}-X_{c^{\prime \prime}}, X_{c^{\prime}}-X_{c^{\prime \prime}}\right) .
\end{aligned}
$$

In the more complicated cases, when the approximation Eq. (39) fails, we should substitute Eq. (41) into Eq. (38)

$$
\begin{aligned}
& \tilde{R}_{2}\left(X_{c}, X_{c^{\prime}}\right) \approx P(c) P\left(c^{\prime}\right) \\
& \times\left\{\prod_{p, l_{p}<l_{c}^{*}} \frac{1-P(p \in c)-P\left(p \in c^{\prime}\right)}{[1-P(p \in c)]\left[1-P\left(p \in c^{\prime}\right)\right]}\right\} \\
& \times \sum_{c^{\prime \prime}} \frac{e^{-\lambda_{c^{\prime \prime}} l_{c^{\prime \prime}}} \tilde{C}_{2}\left(X_{c}-X_{c^{\prime \prime}}, X_{c^{\prime}}-X_{c^{\prime \prime}}\right)}{\prod_{p \in c^{\prime \prime}}\left[1-P(p \in c)-P\left(p \in c^{\prime}\right)\right]},
\end{aligned}
$$

where the product in the braces is assumed to be convergent to some smooth function of both $X_{c}$ and $X_{c}^{\prime}$ but independent of the $l_{c}^{*}$.

The substitution of Eq. (27) into Eq. (42) gives

$$
\begin{aligned}
& \tilde{R}_{2}\left(X, X^{\prime}\right)=\frac{\bar{d}_{\mathrm{ppo}}(X) \bar{d}_{\mathrm{ppo}}\left(X^{\prime}\right)}{\bar{d}_{\mathrm{cpo}}(X) \bar{d}_{\mathrm{cpo}}\left(X^{\prime}\right)} \int_{0}^{\infty} d x^{\prime \prime} \\
& \times \sum_{n^{\prime \prime} \nu^{\prime \prime} \mu^{\prime \prime}} \bar{d}_{\mathrm{cpo}}\left(X^{\prime \prime}\right) \tilde{C}_{2}\left(X-X^{\prime \prime}, X^{\prime}-X^{\prime \prime}\right),
\end{aligned}
$$

where the summation in Eq. (42) is replaced by the integration.

Assuming that the product of the mean densities in Eq. (44) varies slowly on the scale of the $X-X^{\prime}$ dependence of the correlation functions we can apply the normalization conditions Eqs. (7) and (24) to get

$$
\begin{aligned}
& \bar{d}_{\mathrm{ppo}}(X)=\left[\frac{\bar{d}_{\mathrm{ppo}}(X)}{\bar{d}_{\mathrm{cpo}}(X)}\right]^{2} \\
& \times \sum_{n^{\prime} \nu^{\prime} \mu^{\prime}} \int_{0}^{\infty} d x^{\prime} \bar{d}_{\mathrm{cpo}}\left(X^{\prime}\right) \bar{d}_{\mathrm{cpo}}\left(X-X^{\prime}\right),
\end{aligned}
$$

which is satisfied by

$$
\begin{aligned}
& \bar{d}_{\mathrm{ppo}}(X) \propto \frac{1}{x} \frac{e^{-\frac{\mu^{2} a}{2 x}}}{\sqrt{2 \pi x / a}} \frac{e^{-\frac{\nu^{2} b}{2 x}}}{\sqrt{2 \pi x / b}}, \\
& \bar{d}_{\mathrm{cpo}}(X) \propto \gamma \frac{e^{-\frac{\mu^{2} a}{2 x}}}{\sqrt{2 \pi x / a}} \frac{e^{-\frac{\nu^{2} b}{2 x}}}{\sqrt{2 \pi x / b}} .
\end{aligned}
$$

The dependence on $n$ is not known but assumed to be the same for both $\bar{d}_{\mathrm{ppo}}(X)$ and $\bar{d}_{\mathrm{cpo}}(X)$. The parameters $a$ and $b$ here are lengths of the order of the billiard size. The mean density of PPOs Eq. (46) was introduced by Berry and Keating 30 . The numeric tests of Dittrich et al 31 support the distribution Eq. (46). Then the mean density Eq. (47) is the solution of Eq. (45).

Assuming that $\bar{d}_{\mathrm{ppo}}(X) / \bar{d}_{\mathrm{cpo}}(X)=(\gamma x)^{-1}$ for large $x$, we obtain the main result of this section

$$
\tilde{R}(x, y)=\frac{1}{x^{2} \gamma^{2}} \int_{0}^{\infty} d x^{\prime} \tilde{d}_{\mathrm{cpo}}\left(x^{\prime}\right) \tilde{C}\left(x-x^{\prime}, y\right),
$$


which was obtained by the summation on both sides of Eq. (44) over $n, \nu, \mu$ at $n^{\prime}=n, \nu^{\prime}=\nu$, and $\mu^{\prime}=\mu$.

It is instructive to check how Eqs. (38) and (40) work for logarithms of integer and prime numbers. It turns out that Eq. (38) is precisely equivalent to the Hardy - Littlewood conjecture. This can be shown by simple algebra and we put this calculation in Appendix B. At the same time Eq. (40) is equivalent to the smoothed form of the Hardy - Littlewood expression, see Appendix C.

\section{BEHAVIOR OF THE FORM-FACTOR NEAR HEISENBERG TIME.}

For the case of billiards it is convenient to introduce the Heisenberg length $2 \pi \bar{d}(k)$. From Eqs. (10b) and $(25 \mathrm{~d})$ one can see that the diagonal parts of the form-factors are independent of the Heisenberg length, because they are independent of $k$. They are purely classical quantities.

The information about the behavior of the form-factors near the Heisenberg length is hidden in their off-diagonal parts. The off-diagonal parts of the form-factors are not independent; the Fourier transform of Eq. (48) with respect to $y$ gives us

$$
\begin{aligned}
K_{\mathrm{off}}(x, k) & =\frac{1}{4 \pi^{2} \gamma^{2}} \int_{0}^{\infty}\left[K_{\mathrm{diag}}^{\zeta}\left(x^{\prime}\right) K_{\mathrm{off}}^{\zeta}\left(x-x^{\prime}, k\right)\right. \\
& \left.+K_{\mathrm{diag}}^{\zeta}\left(x^{\prime}\right) K_{\mathrm{off}}^{\zeta}\left(-x-x^{\prime}, k\right)\right] d x^{\prime}
\end{aligned}
$$

where we substituted the mean density of CPOs from Eq. 25c) and also used Eqs. (10d) and (25e). In the derivation of Eq. (49), we have assumed that the Heisenberg length is so large that Eqs. (10d), 25c), (25d), and (48) are valid. We also put Eq. (49) in the form which is symmetric with respect to $x$. Equation (49) shows that $K_{\text {off }}(x, k)$ is an analytic function near the Heisenberg length $x \sim 2 \pi \bar{d}(k)$ in agreement with results of Ref. 17. A more detailed comparison of results is possible if we express $K_{\text {off }}^{\zeta}(x, k)$ in terms of $K_{\text {diag }}^{\zeta}(x)$.

The functional equation for the spectral zeta function Eq. (16) results in symmetry properties of the correlation function Eq. (19). The Fourier transform of this equation gives us

$$
K^{\zeta}(x, k)=K^{\zeta}(2 \pi \bar{d}(k)-x, k) .
$$

The derivative with respect to $x$ of both sides of Eq. (50) can be written as

$$
K_{\mathrm{diag}}^{\zeta \prime}(x)+K_{\mathrm{off}}^{\zeta \prime}(x)=-K_{\mathrm{diag}}^{\zeta \prime}(2 \pi \bar{d}-x)-K_{\mathrm{off}}^{\zeta \prime}(2 \pi \bar{d}-x)
$$

where the argument $k$ is omitted at $\bar{d}$ and at $K_{\text {off }}^{\zeta \prime}(x)$. Near the Heisenberg length $K_{\text {diag }}^{\zeta}(x)$ is almost constant and its derivative can be neglected. The same thing is true for $K_{\text {off }}^{\zeta}(x)$ for small $x$. We, therefore, remain with

$$
K_{\mathrm{off}}^{\zeta \prime}(x, k)=-K_{\mathrm{diag}}^{\zeta \prime}(2 \pi \bar{d}(k)-x)
$$

This equation together with Eq. (49) expresses $K_{\text {off }}(x, k)$ in the terms of $K_{\mathrm{diag}}(x), K_{\mathrm{diag}}^{\zeta}(x)$, and $\bar{d}(k)$. Equations (49) and (52) solve the problem of the periodic orbit computation of $K(x, k)$ near $x \sim 2 \pi \bar{d}(k)$.

One can understand the important role of the short composite orbits by looking at another form of Eq. (49) which can be obtained from Eq. (42) by making use of Eqs. (27), (10d) and (25):

$$
K_{\mathrm{off}}(x, k)=\frac{1}{4 \pi \gamma^{2}} \sum_{c} e^{-\lambda_{c} l_{c}} K_{\mathrm{off}}^{\zeta}\left(x-l_{c}, k\right),
$$

valid for $x>0$. Substitution of the integrated Eq. 52 gives

$$
K_{\mathrm{off}}(2 \pi \bar{d}-x)=\sum_{c} \frac{e^{-\lambda_{c} l_{c}}}{4 \pi \gamma^{2}}\left[K_{\mathrm{diag}}^{\zeta}\left(x+l_{c}\right)-\gamma\right]
$$

The function $K_{\text {off }}(2 \pi \bar{d}-x)$ has spikes for the small values of $x$ equal to the length differences of the short CPOs.

The inverse Fourier transform of Eq. (49) would give us the expression for $R_{\text {off }}(\varepsilon, k)$. However, it does not converge, because $K_{\text {off }}^{\zeta}(x, k)$ remains constant when $x$ goes to infinity. The regularization of the inverse Fourier transforms obtained by taking derivatives of Eq. (49) with respect to $x$ and $2 \pi \bar{d}$ gives

$$
R_{\mathrm{off}}(\varepsilon, k)=\frac{\cos (2 \pi \bar{d}(k) \varepsilon)}{2 \pi^{2} \gamma^{2}}\left|C_{\operatorname{diag}}(\varepsilon)\right|^{2}+\bar{d}(k) \delta(\varepsilon) .
$$

This equation, which reproduces the results of Ref. 17, was adopted for the ballistic systems in Ref. 23 and rederived in Ref. 7. see Sec. VI of present work for more detailed discussion.

It is also instructive to check all of our equations for the case when the system exhibits universal behavior. The random matrix theory predicted the correlation functions of the density of states 32 and the spectral determinant 26 to be

$$
\begin{aligned}
& R(\varepsilon, k)=\frac{\cos (2 \pi \bar{d}(k) \varepsilon)-1}{2 \pi^{2} \varepsilon^{2}}+\bar{d}(k) \delta(\varepsilon) . \\
& C(\varepsilon, k)=2 \gamma e^{i \pi \bar{d}(k) \varepsilon} \frac{\sin (\pi \bar{d}(k) \varepsilon)}{\varepsilon}
\end{aligned}
$$

where the latter satisfies the symmetry relation Eq. (19). The commonly accepted form of Eq. (56) can be obtained for $R(\varepsilon, k) /[\bar{d}(k)]^{2}$ and it is $\sin ^{2}(z) / z^{2}$, where $z \equiv \pi \varepsilon \bar{d}(k)$. The universal form-factors are

$$
\begin{aligned}
K(x, k) & =\frac{|x|+2 \pi \bar{d}(k)-|| x|-2 \pi \bar{d}(k)|}{8 \pi^{2}} \\
K^{\zeta}(x, k) & =\gamma \theta(x) \theta(2 \pi \bar{d}(k)-x) .
\end{aligned}
$$

One can note the symmetry of the universal form-factor Eq. (58) with respect to the exchange $x \leftrightarrow 2 \pi \bar{d}(k)$. 
In order to see some kind the of universal length spectrum correlations, let us use the inverse density of states $k_{x}$, which is a purely classical function defined in Eq. (8). The Fourier transforms of Eqs. (58) and (59) with respect to $k$ and the change of sign give

$$
\begin{aligned}
& \tilde{R}(x, y)=\tilde{d}_{\mathrm{ppo}}(x) \frac{1}{x} \int_{0}^{x} d x^{\prime} \frac{\sin \left(k_{x^{\prime}} y\right)}{\pi y} \\
& \tilde{C}(x, y)=\tilde{d}_{\mathrm{cpo}}(x) \frac{\sin \left(k_{x} y\right)}{\pi y} .
\end{aligned}
$$

Both correlation functions satisfy the normalization conditions Eqs. (7) and (24). Equation (61) is the "universal" correlation function of the composite actions. Equation (60) is the essence of the relation Eq. (48) and it is the most general "universal" correlation function of the actions.

\section{DISCUSSION AND SUMMARY}

The principal results of the present work are the statistical relations Eq. (49) or Eq. (53) and the approximate functional equation Eq. (52). The correlation functions and their form-factors depend parametrically on the widths of the averaging windows $\Delta k$ and $\Delta l$. At the same time these parameters do not appear in the statistical relations Eq. (49) and Eq. (54). Therefore, these relations are valid for some values of $\Delta k$ and $\Delta l$ which should be specified.

The probabilistic derivation of Eq. (49) means that $K_{\text {off }}(x, k)$ and $R(\varepsilon, k)$ possess fluctuations. Therefore, one has to choose $\Delta k$ and $\Delta l$ in such a way that that these fluctuations will be smaller than the correlation functions themselves 33

Let us assume that the four-point correlation function of levels has the universal random matrix theory form that is justified for some chaotic systems 34.35. Then one can show, by making use of the geometric representation Fig. 2, that the mean square fluctuations of $R(\varepsilon, k)$ are of the order of $\bar{d}^{3 / 2} \Delta k^{-1 / 2}$.

Let us assume that the two-point correlation function given by Eq. (55) behaves like $1 / \varepsilon^{2}$ for $\varepsilon \bar{d}(k) \gg 1$. Then it becomes of the order of $\Delta l^{2}$ near $\varepsilon \sim 1 / \Delta l$, which is the maximal value of $\varepsilon$ where the definition Eq. (2) is meaningful. Therefore the condition that the fluctuations of $R(\varepsilon, k)$ are smaller than $R(\varepsilon, k)$ itself is

$$
\Delta l^{2} \gg \frac{\bar{d}^{3 / 2}}{\Delta k^{1 / 2}} \text {. }
$$

This inequality, together with Eqs. (13) and (14), restricts the choice of $\Delta k$ and $\Delta l$ strongly.

The inequalities Eq. (14) taken near the Heisenberg length become the approximate equation relating $\Delta l$ to $\Delta k$. We can substitute $\Delta l(\Delta k)$ into Eq. (62) and obtain

$$
\Delta k^{5} \gg \frac{\bar{d}^{3}}{\bar{d}^{4}}
$$

Let $\mathcal{N}(k)$ be a function counting energy levels with $k_{n}<$ $k$. One can apply the approximation $\bar{d}^{\prime}(k) \approx \bar{d}^{2}(k) / \mathcal{N}(k)$ which is valid for generic system. The counting function can be substituted into Eq. (63), and it gives the widths of the averaging window measured in the number of energy levels

$$
\Delta \mathcal{N} \gg \mathcal{N}^{4 / 5}
$$

This inequality has to be satisfied together with $\Delta \mathcal{N} \ll$ $\mathcal{N}$. Therefore the numeric check of relations like Eqs. (54) and (55) is difficult. One has to take at least $\mathcal{N} \sim 10^{10}$ and average over $\Delta \mathcal{N} \sim 10^{9}$ levels in order to see nonuniversal features predicted by Eqs. (54) and (55). The two-point correlation function has to be evaluated for $\varepsilon$ in the range from zero to $\bar{d} / \Delta l \sim \mathcal{N} / \Delta \mathcal{N} \sim 10$ level spacings. This estimate also shows that

$$
\Delta k \Delta l \sim \frac{\Delta \mathcal{N}^{2}}{\mathcal{N}} \gg 1
$$

and the definitions of the correlation functions Eqs. (2) and (18) are justified.

The basic object characterizing a chaotic dynamical system is the set of mixing rates. They show how fast the density-density correlations decay. These rates are zeros of the so-called classical zeta function, see e.g. Ref 36, which is approximately $C_{\text {diag }}(i s)$, see Ref. 7. The functional equation in the form Eq. (52) implies

$$
\varepsilon C_{\mathrm{off}}(\varepsilon, k)=e^{-2 \pi i \bar{d}(k) \varepsilon}\left[\varepsilon C_{\mathrm{diag}}(\varepsilon)\right]^{*},
$$

where $\varepsilon C_{\text {diag/off }}$ is defined as the inverse Fourier transform of $K_{\mathrm{diag} / \mathrm{off}}^{\zeta \prime}$. Therefore $C_{\mathrm{off}}(\varepsilon, k)$ is the classical zeta-function modulated by quantum oscillations. This function contains the information not only on the mixing properties of the billiard, but also on quantum recurrence.

The association of $C_{\text {diag }}(i s)$ with the classical or Ruelle zeta function is valid in the same approximation as Eq. (55) was derived. Therefore, Eq. (55) reproduces exactly results of Ref. 23, for the case of the system with broken time reversal symmetry. The result of Ref. 7 goes beyond the range of lengths given by Eq. (11). One has to make the replacement in Eq. (55)

$$
\left|C_{\operatorname{diag}}(\varepsilon)\right|^{2} \rightarrow[2 \pi \bar{d}(k) \gamma]^{2}\left\langle\frac{\zeta(k+\varepsilon / 2) \zeta^{*}(k-\varepsilon / 2)}{\zeta^{*}(k+\varepsilon / 2) \zeta(k-\varepsilon / 2)}\right\rangle_{\operatorname{diag}}
$$

and use the product Eq. (17) truncated near the Heisenberg length $l_{p} \leq 2 \pi \bar{d}(k)$ in order to obtain the more accurate results of Ref. 7. We performed the more accurate computation for the prime numbers, see Appendix B, and obtained the precise form of the Hardy - Littlewood conjecture, which is equivalent to the replacement Eq. (67) in Eq. (55).

All calculations in this work were done for chaotic billiards, where the action of orbit $p$ has the very simple 
form $S_{p}=\hbar k l_{p}$. The present theory can be generalized for other types of dynamical chaotic systems. The only problem is that the correlation function of actions $\left\langle\delta\left(x-S_{p}\right) \delta\left(x^{\prime}-S_{p^{\prime}}\right)\right\rangle$ has to be defined by averaging over the constant mean period $\partial S_{p} / \partial E+\partial S_{p^{\prime}} / \partial E$. The justification of such procedure was discussed in Refs. 8 , 9.

The result of the present work cannot be applied to the systems which possess time reversal symmetry. In this case we should compute $\tilde{C}_{2}\left(X ; X^{\prime}\right)$ or $\tilde{R}_{2}\left(X ; X^{\prime}\right)$ for $\mu \neq \mu^{\prime}$, i.e. the action correlations of orbits with different winding numbers. However, the results presented here can be generalized for a system having discrete symmetriest 10 , see also a discussion of this problem by Leyvraz and Seligman 37 and Agam et a 38 . In the case of the discrete symmetry of the system, one should compute the multiplicities of the periodic orbits and correlations of these multiplicities in order to obtain the spectral statistics. Probabilistic number theory methods can be used again for this purpose as in the case of the modular group. 39

In summary we have shown that the functional equation for the spectral determinant implies the action correlations. Quantum-classical time scale separation for systems with broken time reversal symmetry allows us to compute all spectral and action correlation functions beyond their universal random-matrix theory shapes. One of the central technical points in the present work is the derivation of the Hardy - Littlewood conjecture of primenumber correlations in such a way that it can be used for actions of a dynamical system.

\section{ACKNOWLEDGMENTS}

It is my pleasure to thank U. Smilansky, D. Cohen, Z. Rudnik, H. Primack, E. Bogomolny, IU. M. Galperin, M. Berry and J. P. Keating for very useful discussions. This work was supported by Israel Science Foundation.

\section{APPENDIX A: NORMALIZATION OF THE CORRELATION FUNCTIONS.}

The normalization conditions Eqs. (7) and (24) are generic for the correlation function and we expect that $\sum_{c^{\prime}}\left[P(c) P\left(c^{\prime}\right)-P\left(c, c^{\prime}\right)\right]=P(c)$ and $\sum_{c^{\prime}}\left[P\left(c^{\prime \prime} \in\right.\right.$ c) $\left.P\left(c^{\prime \prime} \in c^{\prime}\right)-P\left(c^{\prime \prime} \in c \cap c^{\prime \prime} \in c^{\prime}\right)\right]=P\left(c^{\prime \prime} \in c\right)$. The sum over $c^{\prime}$ in Eq. (38) leads to

$$
P(c) \approx \sum_{c^{\prime \prime}} P\left(c^{\prime \prime} \in c\right) \prod_{p \notin c^{\prime \prime}, l_{p}<l_{c}^{*}}[1-2 P(p \in c)] .
$$

We can represent $P\left(c^{\prime \prime} \in c\right)$ as a product, and then

$$
\begin{aligned}
P(c) & \approx \prod_{l_{p}<l_{c}^{*}}[1-2 P(p \in c)] \sum_{c^{\prime \prime}} \prod_{p \in c^{\prime \prime}, l_{p}<l_{c}^{*}} \frac{P(p \in c)}{1-2 P(p \in c)} \\
& \approx \prod_{l_{p}<l_{c}^{*}}[1-2 P(p \in c)]\left[1+\frac{P(p \in c)}{1-2 P(p \in c)}\right] \\
& =\prod_{l_{p}<l_{c}^{*}}[1-P(p \in c)]
\end{aligned}
$$

in agreement with Eq. (31).

\section{APPENDIX B: CORRELATIONS OF PRIMES OBTAINED FROM CORRELATIONS OF INTEGERS.}

We can demonstrate the relation between the correlation functions of composite and primitive actions using the example of integer and prime numbers. Let us consider the prime number $p$ as a PPO of length $\log (p)$ and the integer number $n$ as a CPO of length $\log (n)$. This "CPO" may contain repetitions of "PPO", but this is not important for large numbers.

Saying that the "CPO" $m$ "is a part of" "CPO" $n$ means that $m$ is a divisor of $n$, and we will write $m \mid n$. This notation allows us to rewrite Eq. (38) as

$$
\begin{aligned}
& P(n) P\left(n^{\prime}\right)-P\left(n, n^{\prime}\right) \\
& =\sum_{m}\left\{\left[P(m \mid n) P\left(m \mid n^{\prime}\right)-P\left(m|n \cap m| n^{\prime}\right)\right]\right. \\
& \left.\times \prod_{p \nmid m}\left[1-P(p \mid n)-P\left(p \mid n^{\prime}\right)\right]\right\},
\end{aligned}
$$

where $m$ runs over integers which do not contain powers of primes. Here $P(n)$ is the probability to find a prime number, and $P\left(n, n^{\prime}\right)$ is the probability to find pair of primes, see Refs. 40, 41 for precise definitions.

The derivation of the Hardy - Littlewood expression is based on the probability $€$ that $m$ is a divisor of $n$

$$
P(m \mid n)=\frac{1}{m} .
$$

Our derivation is based on the probability to find a common divisor

$$
P\left(m|n \cap m| n^{\prime}\right)=\frac{1}{m} \sum_{l \neq 0} \delta_{n-n^{\prime}-m l},
$$

where the Kroneker $\delta$ symbol in the right hand side is equal to one for $n=n^{\prime}-m l$ and equal to zero otherwise. It carries information about correlations of integers, because if we found with probability $1 / m$ that $n$ is multiple of $m$, then all integers $n^{\prime}=n+m l$ are also multiples of $m$ with the probability 1 .

Substituting the probability Eq. (B3) into Eq. (B1) we obtain 


$$
\begin{aligned}
& P(n) P\left(n^{\prime}\right)-P\left(n, n^{\prime}\right) \\
& =\sum_{m}\left\{\left[\frac{1}{m^{2}}-\frac{1}{m} \sum_{l \neq 0} \delta_{n, n^{\prime}-m l}\right] \prod_{p \nmid m}\left[1-\frac{2}{p}\right]\right\},
\end{aligned}
$$

and the right hand side of this equation is zero if $m$ is odd. Indeed, if $m$ is odd then $p=2$ is not a divisor of $m$ and $1-2 / p$ gives zero. The density of prime numbers, $P(n)=$ $\prod_{p}(1-1 / p)$, must follow the prime number theorem and this is the criterion for choice of the upper limit in the products over primes.

The next step, which is not necessary, is to separate the smooth part from the part containing correlations

$$
P\left(n, n^{\prime}\right)=\sum_{m \mid\left(n-n^{\prime}\right)} \frac{1}{m} \prod_{p \nmid m}\left[1-\frac{2}{p}\right],
$$

and this expression is non-zero only if $n-n^{\prime}$ is even. Only terms with even $m$ contribute and the term with $m=2$ is always present. We can, therefore, extract the factor 2 from all terms, and we obtain for even $n-n^{\prime}$

$$
\begin{aligned}
P\left(n, n^{\prime}\right) & =\frac{1}{2} \prod_{p \geq 3}\left[1-\frac{2}{p}\right] \\
& +\sum_{m \mid\left(n-n^{\prime}\right)} \frac{1}{2 m} \prod_{p \nmid m, p \geq 3}\left[1-\frac{2}{p}\right],
\end{aligned}
$$

where $m$ is odd and does not contain powers of primes. We can rewrite the sum as a product

$$
P\left(n, n^{\prime}\right)=\frac{1}{2} \prod_{p \geq 3}\left[1-\frac{2}{p}\right] \prod_{p \mid\left(n-n^{\prime}\right), p \geq 3} \frac{p-1}{p-2} .
$$

This product contains the correct enhancement 42 of the probability to find a pair of primes $n, n^{\prime}$ by the factor $(p-1) /(p-2)$ per each prime, which is divisor of $n-n^{\prime}$.

A similar computation shows that

$$
\sum_{m} \frac{1}{m^{2}} \prod_{p \nmid m}\left(1-\frac{2}{p}\right)=\prod_{p}\left(1-\frac{1}{p}\right)^{2}=P(n) P\left(n^{\prime}\right) .
$$

Therefore we have shown that Eq. (B5) follows Eq. (B)

The product Eq. (B7) diverges to zero for large $n$. For this reason the probability $P\left(n, n^{\prime}\right)$ is divided by $P(n)=$ $\prod_{p}(1-1 / p)$, see Ref. 43, Chap. Postscript on prime-pairs.

$$
\frac{P\left(n, n^{\prime}\right)}{P(n) P\left(n^{\prime}\right)}=2 \prod_{p \geq 3} \frac{1-2 / p}{(1-1 / p)^{2}} \prod_{p \mid\left(n-n^{\prime}\right)}\left[1+\frac{1 / p}{1-2 / p}\right] .
$$

This correlation function contains only the convergent products and it allows one to compute statistics of the zeros of Riemann zeta function with great accuracy.

\section{APPENDIX C: SMOOTHED CORRELATION FUNCTION OF PRIMES.}

The smoothed form of the correlation function of prime numbers is valid for $\left|n-n^{\prime}\right| \gg 1$, and it can be obtained directly from Eq. (40)

$$
1-\frac{P\left(n, n^{\prime}\right)}{P(n) P\left(n^{\prime}\right)} \approx \sum_{m}\left[\frac{1}{m^{2}}-P\left(m|n \cap m| n^{\prime}\right)\right] .
$$

In this equation we used the probabilities of finding the prime number $P(n)$ and the probability of finding the pair of prime numbers $P\left(n, n^{\prime}\right)$, see Refs. 40, 41, and we substituted $1 / m^{2}$ instead of $P(m \mid n) P\left(m \mid n^{\prime}\right)$.

The smoothing of the probability to have a common divisor can be done in a number of different ways. We suggest that the simplest one is

$$
\frac{1}{m} \int_{-m / 2}^{m / 2} P\left(m|n \cap m| n^{\prime}\right) d n^{\prime}=\frac{1}{m^{2}} \theta\left(\left|n-n^{\prime}\right|-\frac{m}{2}\right) .
$$

The summation over $m$ in Eq. (C1) can be replaced by the integration and we obtain

$$
\begin{aligned}
\frac{P\left(n, n^{\prime}\right)}{P(n) P\left(n^{\prime}\right)} & \approx 1-\int^{\infty} d m \frac{1-\theta\left(\left|n-n^{\prime}\right|-m / 2\right)}{m^{2}} \\
& \approx 1-\frac{1}{2\left|n-n^{\prime}\right|} .
\end{aligned}
$$

The choice of the lower limit in the integral is not important, because the integration is performed from $2\left|n-n^{\prime}\right|$. This result is the leading order expansion of the correlation fynction in $\left|n-n^{\prime}\right|^{-1}$ and it coincides with Keating's result. 40

The probability of finding a prime number can be computed from Eq. (C3) by application of the normalization condition Eq. (7)

$$
\begin{aligned}
\frac{1}{P(n)} & =\sum_{n^{\prime}}\left\{1-\frac{P\left(n, n^{\prime}\right)}{P(n) P\left(n^{\prime}\right)}\right\} \\
& \approx \sum_{\left|n-n^{\prime}\right|<n} \frac{1}{2\left|n-n^{\prime}\right|} \approx \log (n)
\end{aligned}
$$

according to the prime number theorem 44.43 .

\footnotetext{
${ }^{1}$ M. V. Berry, F.R.S., Proc. R. Soc. Lond. A 413, 183 (1987).

${ }^{2}$ O. Bohigas, M. J. Giannonni, and C. Schmit, Phys. Rev. Lett. 52, 1 (1984).

${ }^{3}$ M. V. Berry, F.R.S., in Quantum Chaos, edited by G. Casati and B. Chirikov (Cambridge university press, Cambridge, 1995), Chap. Semiclassical theory of spectral rigidity, p. 319, [Proc. R. Soc. London, A400, 229(1985)].
} 
${ }^{4}$ M. G. Gutzwiller, in Chaos in Classical and Quantum Mechanics, Vol. 1 of Interdisciplinary Applied Mathematics, edited by F. John (Springer-Verlag, New York, 1990).

${ }^{5}$ J. H. Hannay and A. M. Ozorio De Almeida, J. Phys. A: Math. Gen. 17, 3429 (1984).

${ }^{6}$ We assume that the density - density correlations decay as $e^{-t / \tau_{\text {erg }}}$ or faster. For simple chaotic billiards this time is approximately twice inverse Lyapunov exponent, for more complicated systems it is the time of the diffusion through the system., .

${ }^{7}$ E. B. Bogomolny and J. P. Keating, Phys. Rev. Lett. 77, 1472 (1996).

${ }^{8}$ N. Argaman, F.-M. Dittes, E. Doron, J. P. Keating, A. Yu. Kitaev, M. Sieber, and U. Smilansky, Phys. Rev. Lett. 71, 4326 (1993).

${ }^{9}$ D. Cohen, chao-dyn/9612014.

${ }^{10}$ D. Cohen, H. Primack and U. Smilansky, To be published in Adv. Phys. (1997), chao-dyn/9708017.

${ }^{11}$ A. Voros, J. Phys. A 21, 685 (1988).

12 M. V. Berry and J. P. Keating, J. Phys. A 23, 4839 (1990).

13 S. Kettemann, D. Klakow and U. Smilansky, J. Phys. A 30, 3643 (1997).

14 J. P. Keating, Proc. R. Soc. Lond. A 436, 99 (1992).

${ }^{15}$ R. Balian and C. Bloch, Ann. Phys. 85, 514 (1974).

${ }^{16}$ G. H. Hardy and J. E. Littlewood, Acta Mathematica 44, 1 (1922), [Conjecture B, p. 42].

17 A. V. Andreev and B. L. Altshuler, Phys. Rev. Lett. 75, 902 (1995).

${ }^{18}$ K. B. Efetov, Adv. Phys. 32, 53 (1983).

19 B. L. Altshuler and B. I. Shklovskii, Sov. Phys. JETP 64, 127 (1986), [Zh. Eksp. Teor. Fiz. 91, 220 (1986)].

${ }^{20}$ N. Argaman, Y. Imry and U. Smilansky, Phys. Rev. B 47, 4440 (1993).

${ }^{21}$ B. A. Muzykantskii and D. E. Khmelnitskii, JETP Lett. 62, 76 (1995), [Pis'ma Zh. Eksp. Teor. Fiz. 62, 68(1995)].

${ }^{22}$ E. M. Lifshitz and L. P. Pitaevsky, Physical Kinetics, Vol. 10 of Landau and Lifshitz: course of theoretical physics (Pergamon Press, Oxford, 1980), Chap. I., §19.

23 O. Agam, B. L. Altshuler and A. V. Andreev, Phys. Rev. Lett. 75, 4389 (1995).

${ }^{24}$ A. V. Andreev, B. D. Simons, O. Agam, and B. L. Altshuler, Nucl. Phys. B 482, 536 (1996), cond-mat/9605204.

25 A. V. Andreev and B. D. Simons, Phys. Rev. Lett. 75, 2304 (1995).

${ }^{26}$ F. Haake, M. Ku's, H.-J. Sommers, H. Schomerus and K. Zyczkowski, J. Phys. A 29, 3641 (1996).

${ }^{27}$ E. Bogomolny, O. Bohigas, and P. Leboeuf, J. Stat. Phys. 85, 639 (1996).

${ }^{28}$ U. Smilansky, in Mesoscopic quantum physics, edited by E. Akkermans, G. Motambaux, J.-L. Pichard and J. ZinnJustin (Elsevier Science B. V., Amsterdam, 1995), Chap. 7. Semiclassical quantization of chaotic billiards., p. 373.

${ }^{29}$ R. Aurich and F. Steiner, Phys. Rev. A 46, 771 (1992).

${ }^{30}$ M. V. Berry and J. P. Keating, J. Phys. A 27, 6167 (1994).

31 T. Dittrich, B. Mehlig, H. Schanz, and U. Smilansky, to be published (1997).

32 M. L. Mehta, Random Matrices, 2nd ed. (Academic Press, San Diego, 1990).

${ }^{33}$ R. E. Prange, Phys. Rev. Lett. 78, 2280 (1997).

${ }^{34}$ E. B. Bogomolny and J. P. Keating, Nonlinearity 8, 1115
(1995).

${ }^{35}$ P. Shukla, Phys. Rev. E 55, 3886 (1997).

${ }^{36}$ R. Artuso, E. Aurell and P. Cvitanović, Nonlinearity 3, 325 (1990).

${ }^{37}$ F. Leyvraz and T. H. Seligman, Phys. Rev. Lett. 79, 1778 (1997).

${ }^{38}$ O. Agam, A. V. Andreev, B. D. Simons, and B. L. Altshuler, Phys. Rev. Lett. 79, 1779 (1997).

${ }^{39}$ E. Bogomolny, F. Leyvraz and C. Schmit, Commun. Math. Phys. 176, 577 (1996).

${ }^{40}$ J. P. Keating, in Proceedings of Enrico Fermi International School of Physics, Course CXIX (North-Holland, Amsterdam, 1992), Chap. The Riemann zeta-function and quantum chaology, p. 145.

${ }^{41}$ M. Kac, Probability and related topics in Physical Sciences (Interscience, London, 1959), Chap. I. Nature of probabilistic reasoning, p. 12.

${ }^{42}$ Cherwell, Quart. J. Math. (Oxford) 17, 46 (1946).

${ }^{43}$ G. H. Hardy and E. M. Wright, An introduction to the theory of numbers (Oxford University Press, London, 1956).

${ }^{44}$ H. M. Edwards, Riemann's Zeta Function (Academic Press, New York, 1974), Vol. 1. 